\title{
\begin{tabular}{l|l} 
MitTraries & DSpace@MIT
\end{tabular}
}

\author{
MIT Open Access Articles
}

\section{Influence of a Metallic Shield on RF-Induced Heating of a Lead with Straight and Helical Wires}

The MIT Faculty has made this article openly available. Please share how this access benefits you. Your story matters.

Citation: Kozlov, Mikhail et al. "Influence of a Metallic Shield on RF-Induced Heating of a Lead with Straight and Helical Wires." 2019 IEEE MTT-S International Conference on Numerical Electromagnetic and Multiphysics Modeling and Optimization (NEMO), May 2019, Boston, MA, October 2019. (C) 2019 IEEE

As Published: $10.1109 /$ nemo.2019.8853734

Publisher: IEEE

Persistent URL: https://hdl.handle.net/1721.1/130947

Version: Author's final manuscript: final author's manuscript post peer review, without publisher's formatting or copy editing

Terms of use: Creative Commons Attribution-Noncommercial-Share Alike 


\section{Influence of a Metallic Shield on RF-Induced Heating of a Lead with Straight and Helical Wires}

\author{
Mikhail Kozlov \\ Max Plank Inst. for Human Cognitive and \\ Brain Sciences \\ Leipzig, Germany \\ kozlov@cbs.mpg.de
}

\author{
Wolfgang Kainz \\ U.S. FDA \\ Silver Spring, MD, USA \\ wolfgang.kainz@fda.hhs.gov
}

\author{
Luca Daniel \\ Massachusetts Institute of Technology \\ Cambridge, MA, USA \\ luca@mit.edu
}

\begin{abstract}
We present results including a lead electromagnetic model (LEM), power deposition, and RF-induced heating for a set of single electrode leads with straight and helical wires, as regular unshielded lead, and leads with a metallic shield. Our results were obtained numerically, employing 3D electromagnetic and thermal co-simulations at $128 \mathrm{MHz}$. We observe that the metallic shield raises the LEM calibration factor and thereby it increases the RF-induced heating of the lead with helical wires, a circumstance that previously had mostly been neglected.
\end{abstract}

Keywords- electromagnetics, $R F$ safety, heating, active implantable medical devices, transfer functions

\section{INTRODUCTION}

Tissue damage near the lead tip of an active implantable medical device (AIMD), or near the edge of a passive implant can occur in a patient undergoing magnetic resonance imaging (MRI). This is due to tissue heating produced by radio frequency $(\mathrm{RF})$ induced power deposition [1-4].

Domain decomposition is a common technique involving splitting a solution of a complex problem into a set of substantially simpler sub-tasks. For RF-induced heating, one possible domain decomposition approach consists of separating the assessment of the human RF exposure due to an MRI whole-body coil from the assessment of the AIMD RF-induced power deposition due to an incident RF field (as defined in Clause \#8 Tier 3 of ISO/TS 10974:2018 [5]).

The total RF power $(P)$ dissipated around the AIMD lead electrode can be derived integrating the volume loss density (VLD)

$$
P=\int_{H S I V}\left(V L D_{\text {total }}(v)-V L D_{\text {backgnd }}(v)\right) \cdot d v
$$

where: $V L D_{\text {total }}(v)$ considers the lead in place, while $V L D_{\text {backgnd }}(v)$ does not have the lead in place. HSIV is the hot spot integration volume. The volume loss density can be estimated using $\sigma \cdot|E(v)|^{2}$, where $\sigma$ is electrical conductivity of the surrounding medium and $E$ is the electrical field.

The net temperature increase $\left(\Delta T_{\mathrm{p}}\right)$ as well as $P$, can also be estimated using the lead electromagnetic model (LEM) as defined in ISO/TS 10974:2018 [5]

$$
\begin{aligned}
& \Delta T_{p}=A_{T p} \cdot\left|\int_{0}^{L} S(l) \cdot E_{\mathrm{tan}}(l) \cdot d l\right|^{2}, \\
& P=A \cdot\left|\int_{0}^{L} S(l) \cdot E_{\mathrm{tan}}(l) \cdot d l\right|^{2}
\end{aligned}
$$

where: $A_{T \mathrm{p}}$ and $A$ are the calibration factors of the LEM for $P$ and $\Delta T_{\mathrm{p}}$, respectively, $S(l)$ is the transfer function (TF), $E_{\tan }(l)$ is an incident tangential electric field along the AIMD lead trajectory, and $L$ is the lead length.

Recently, [6] proposed using a metallic shield around the lead to reduce the RF-induced heating. In that approach, the metallic shield was in direct contact with the surrounding media, a situation that could generate bio-compatibility issues for real world leads, because usually an AIMD lead is coated by an insulator material in order to avoid bio-compatibility issues.

The main goals of our study were: $(i)$ to evaluate the influence of the metallic shield on $P, \Delta T_{p}$, and on the LEM for different lead properties; (ii) to evaluate the consequences of a shield that was with, or without contact to the surrounding medium in close proximity to the center electrode.

\section{Methods AND PROCEDURES}

Our set of twelve generic leads was formed by leads with straight and helical titanium alloy wires, with and without a titanium alloy shield. The length of the leads was $320 \mathrm{~mm}$. The tip electrode diameter was $0.25 \mathrm{~mm}$ with a length of $10 \mathrm{~mm}$. The diameter of the unshielded lead insulation was $1.46 \mathrm{~mm}$. The straight wire diameter was $0.73 \mathrm{~mm}$. A helical structure was a $0.1 \mathrm{~mm} \times 0.1 \mathrm{~mm}$ rectangular wire with a pitch of 0.35 $\mathrm{mm}$ and with an external diameter of $0.9 \mathrm{~mm}$ (Fig. 1a-b). The proximal lead end was capped with an insulator.

The shield and shield insulator outer diameters were 1.56 $\mathrm{mm}$ and $2.5 \mathrm{~mm}$, respectively. An area to provide an electrical contact between shield and surrounding medium was located 3 $\mathrm{mm}$ away from the electrode and was $3 \mathrm{~mm}$ in length (Fig. 1c). The impedance between the inner wire and the external metallic shield was defined as infinite. When evaluating the shielded lead without medium contact to the shield, the contact area was covered by an insulator.

Values of relative permittivity $\left(\varepsilon_{\mathrm{r}}\right)$ of the insulators were 2.7 and 5.5 , respectively. The electric conductivity of the titanium alloy was $\sigma=595238 \mathrm{~S} / \mathrm{m}$, specific heat $C_{p}=526.3 \mathrm{~J} / \mathrm{kg} / \mathrm{K}$, isotropic thermal conductivity $k=6.7 \mathrm{~W} / \mathrm{m} / \mathrm{K}$, and density $\rho=$ $4430 \mathrm{~kg} / \mathrm{m}^{3}$. The insulation material has $\sigma=24 \mu \mathrm{S} / \mathrm{m}, \varepsilon_{\mathrm{r}}=2.7$, $C_{p}=1000 \mathrm{~J} / \mathrm{kg} / \mathrm{K}, k=0.2 \mathrm{~W} / \mathrm{m} / \mathrm{K}$, and $\rho=1350 \mathrm{~kg} / \mathrm{m}^{3}$.

The leads were positioned parallel to the $\mathrm{Z}$ axis in the middle of a box $(600 \mathrm{~mm} \times 400 \mathrm{~mm} \times 2400 \mathrm{~mm})$ surrounded by a perfectly matched layer (PML). The box was filled by a 
medium with electrical properties similar to blood at $128 \mathrm{MHz}$, namely $\varepsilon_{\mathrm{r}}=78$ and $\sigma=1.2 \mathrm{~S} / \mathrm{m}$, and thermal properties: $C_{p}=$ $4181 \mathrm{~J} / \mathrm{kg} / \mathrm{K}, k=0.6 \mathrm{~W} / \mathrm{m} / \mathrm{K}$, and $\rho=1001 \mathrm{~kg} / \mathrm{m}^{3}$ (Fig. 1d).

Four antennas located along one yz side of the box generated the EM field at $128 \mathrm{MHz}$ as in our previous study [7]. To acquire $E_{\tan }(l)$ along the lead trajectory 3D EM simulations were done without the lead in place. The heterogeneous $E_{\tan }(l)$ was equal to the $\hat{z}$-component of the electric field at the location of the lead.

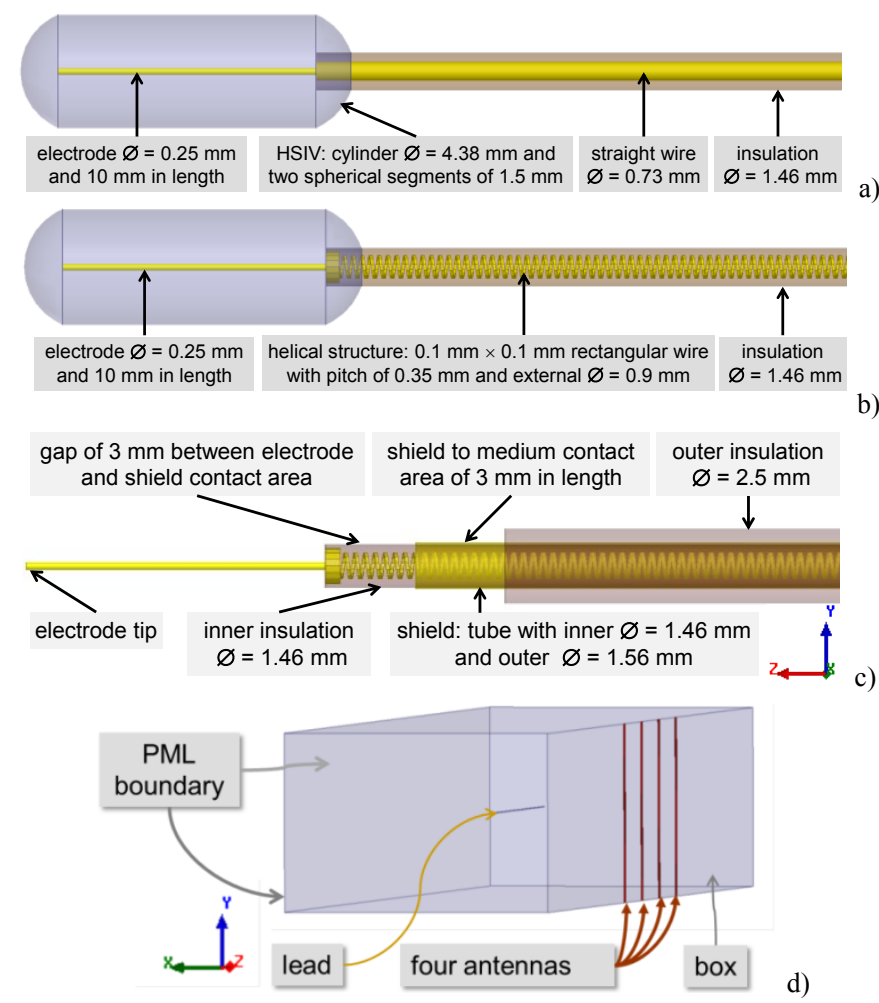

Fig. 1. a) Close-up view of unshielded straight lead geometry. b) Close-up view of unshielded straight lead geometry. c) Close-up view of the shielded lead geometry near the electrode. d) 3D electromagnetic simulation setup.

The transfer function $S(l)$ for each lead was obtained at 161 equidistant points, using the reciprocity approach outlined in [8]. In order to enable a comparison between different cases, $S(l)$ was normalized so that $\int_{0}^{L}\|S(l)\| \cdot d l=1$. The dissipated power $P$ was calculated for all $E_{\tan }(l)$ with the HSIV defined in Fig. 1a and in (1), using 3D EM simulations with the leads in place. $\Delta T_{\mathrm{p}}$ was analyzed for the electrode tip face (Fig. 1c) as the net temperature increase after 60 seconds of continuous excitation. The time interval of 60 second was selected in order to avoid substantial influence of the medium thermal conductivity onto the thermal results.

The calibration factors of the LEM were derived from a linear regression analysis of $P$ and $\Delta T_{\mathrm{p}}$ versus the LEM predicted results

$$
L E M=\left|\int_{0}^{L} S(l) \cdot E_{\tan }(l) \cdot d l\right|^{2} .
$$

The 3D EM simulations were performed at $128 \mathrm{MHz}$ using ANSYS HFSS (ANSYS, Canonsburg, PA, USA). VLD and surface loss density from our 3D EM simulations were scaled with a scaling factor so that the maximum temperature rise in the thermal domain was less than $10^{\circ} \mathrm{C}$ for 15 minutes of continuous excitation. The thermal simulations were performed in ANSYS Non-Linear Thermal platform (ANSYS, Canonsburg, PA, USA). For our subsequent analysis the results of the thermal simulations were scaled to the $E_{\mathrm{tan}}(l)$ generated in the phantom without the lead in place. The tetrahedron mesh and the solver convergence setups were the same as in our previous study [7].

\section{RESULTS AND DISCUSSION}

The lead center wire geometry, (i.e., straight vs. helical) affected by a noticeable factor of two the $S(l)$ of the unshielded leads (Fig. 2a-b). Instead, the $S(l)$ of the shielded lead were very similar (difference up to $10 \%$ ) to the $S(l)$ of the unshielded leads with a straight wire (Fig. 2a,c-f) with corresponding insulator $\varepsilon_{\mathrm{r}}$.
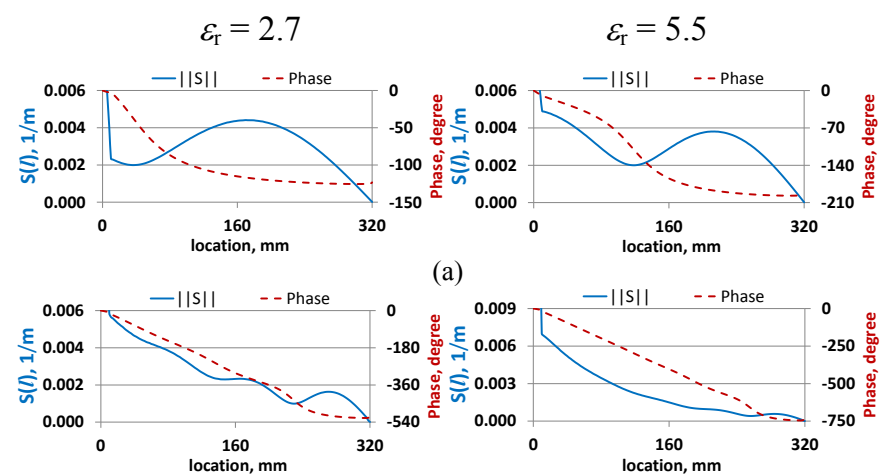

(a)
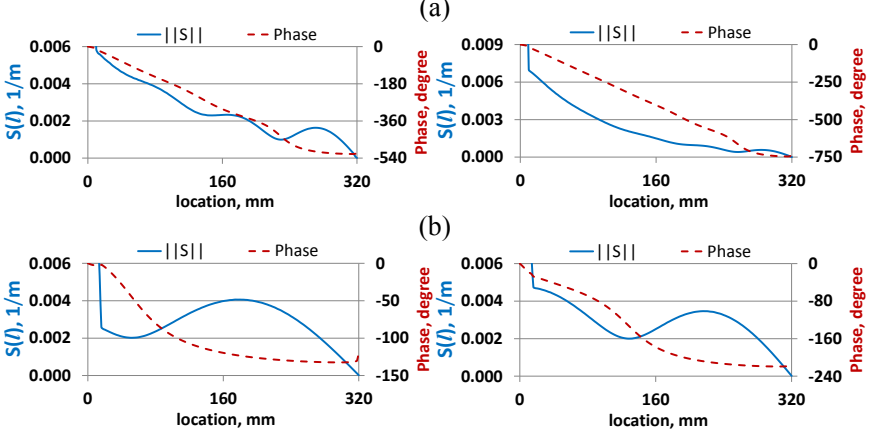

(b)
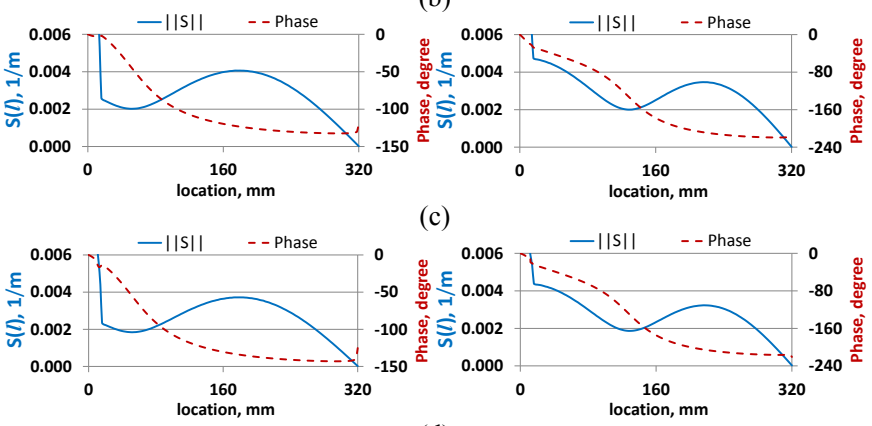

(c)

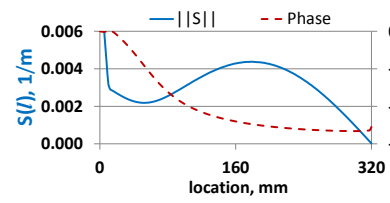

(d)
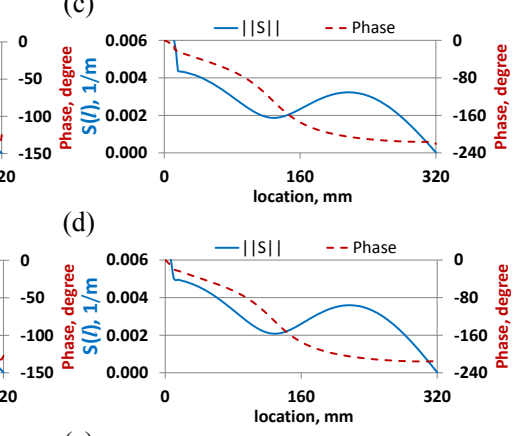

(e)
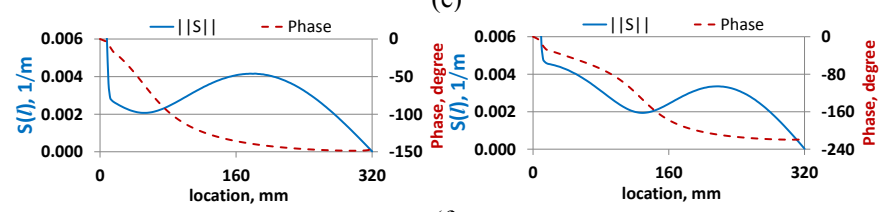

(f)

Fig. 2. Amplitudes and phases of $S(l)$ for different lead geometries and insulator $\varepsilon_{\mathrm{r}}$ : a) unshielded straight lead, b) unshielded helical lead, c) shielded straight lead with medium contact of the shield, d) shielded helical lead with medium contact of the shield, e) shielded straight lead without medium contact of the shield, f) shielded helical lead without medium contact of the shield. 

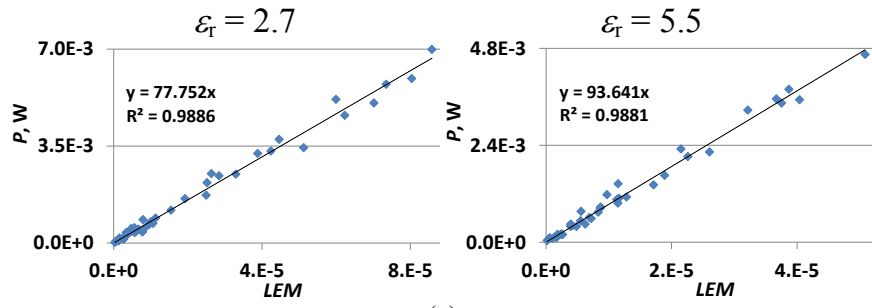

(a)

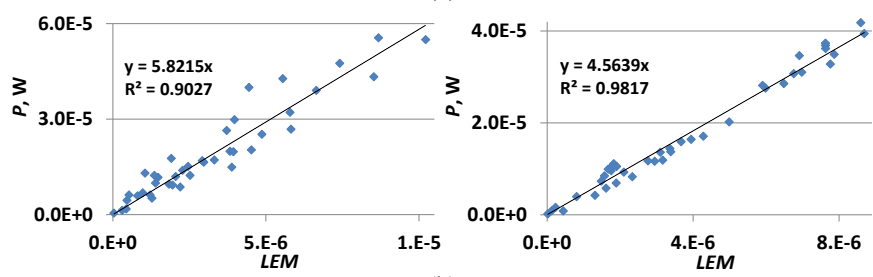

(b)

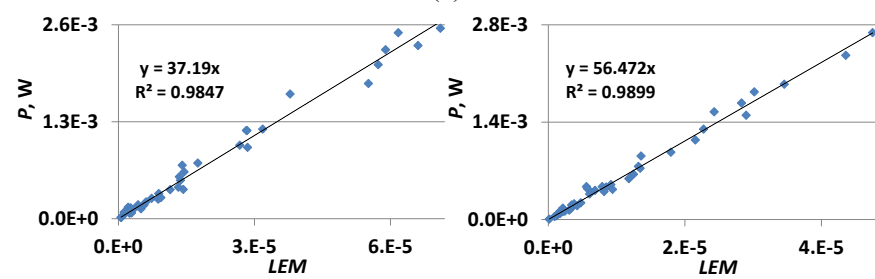

(c)

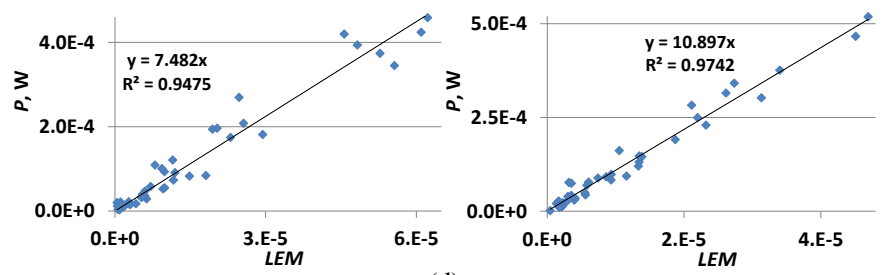

(d)

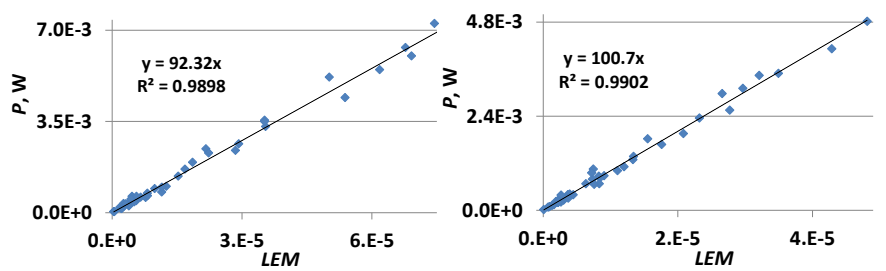

(e)
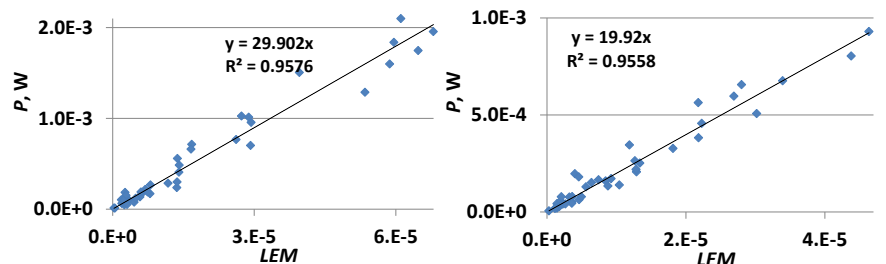

(f)

Fig. 3. The regression analysis of $P$ versus $L E M$ for different lead geometries and insulator $\varepsilon_{\mathrm{r}}$ : a) unshielded straight lead, b) unshielded helical lead, c) shielded straight lead with medium contact of the shield, d) shielded helical lead with medium contact of the shield, e) shielded straight lead without medium contact of the shield, f) shielded helical lead without medium contact of the shield.

Despite the similar $S(l)$ of the unshielded and shielded straight lead, the calibration factor $A$ significantly depends on the electrical contact of the shield with the medium. Without electrical contact, $A$ and $P$ of the shielded straight lead were similar (difference up to $20 \%$ for $A$, and $12 \%$ for $P$, respectively) to the unshielded straight lead. The contact with the surrounding medium reduced $P$ of shielded straight lead by a factor of 2.7 and 1.7 (Fig. 3) for an insulator $\varepsilon_{\mathrm{r}}$ of 2.7 and 5.5, respectively.

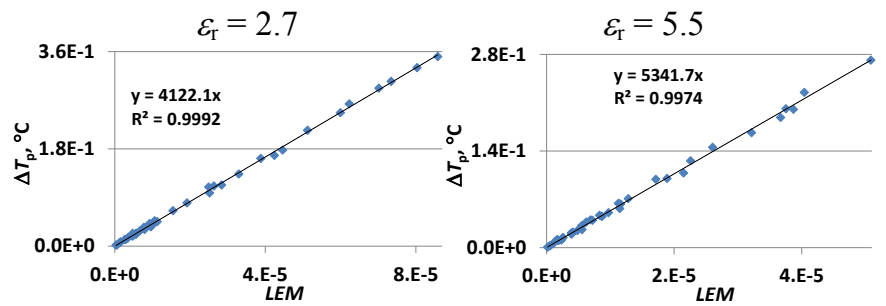

(a)

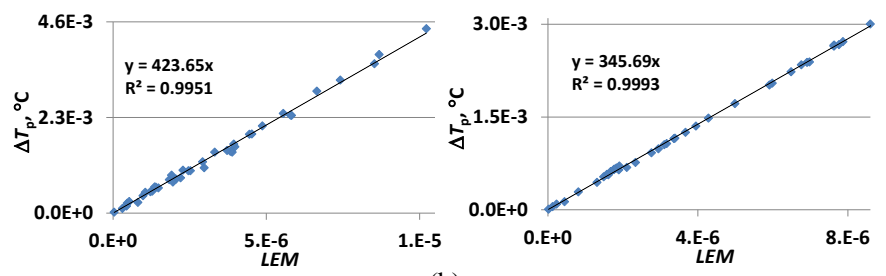

(b)

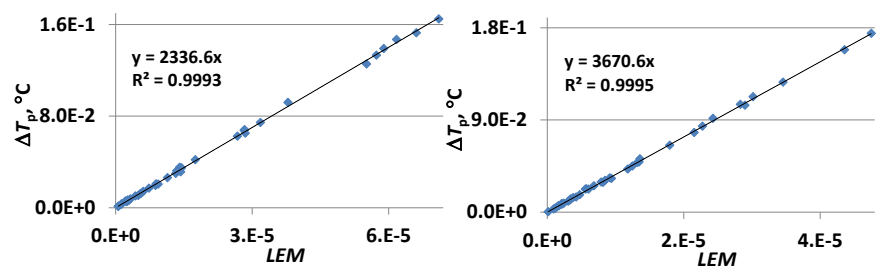

(c)

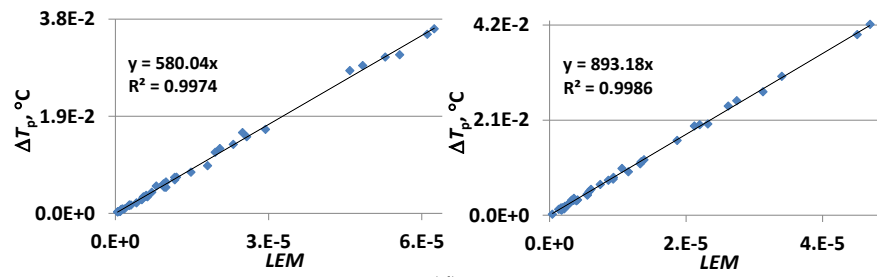

(d)

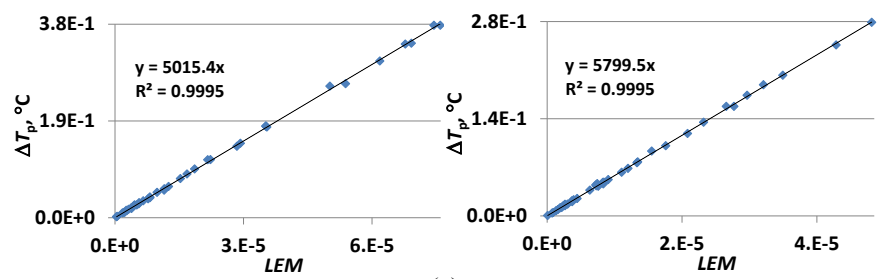

(e)

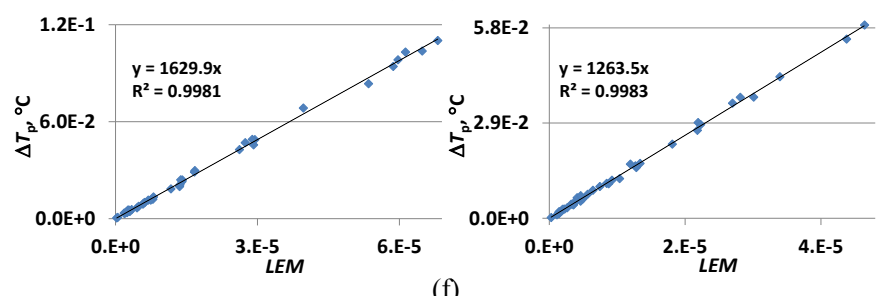

(f)

Fig. 4. The regression analysis of $\Delta T_{p}$ versus $L E M$ for different lead geometries and insulator $\varepsilon_{\mathrm{r}}$ : a) unshielded straight lead, b) unshielded helical lead, c) shielded straight lead with medium contact of the shield, d) shielded helical lead with medium contact of the shield, e) shielded straight lead without medium contact of the shield, f) shielded helical lead without medium contact of the shield. 
Without and with medium contact of the shield $\Delta T_{\mathrm{p}}$ were significantly higher for the shielded helical lead than for unshielded helical lead (specifically, higher by a factor of 8.2 and 14 for an insulator with $\varepsilon_{\mathrm{r}}=2.7$ and $\varepsilon_{\mathrm{r}}=5.5$, respectively). For the shielded helical leads the contact with the medium reduced both the scaling factor and $\Delta T_{\mathrm{p}}$ by a factor of 3.1 and 1.4 (Fig. 4) for insulator $\varepsilon_{\mathrm{r}}$ of 2.7 and 5.5, respectively.

We observed a different dependence of $P$ and $\Delta T_{\mathrm{p}}$ on the insulator relative permittivity $\varepsilon_{\mathrm{r}}$, wire geometry, and metallic shield. For the helical lead, the metallic shield resulted in approximately one order of magnitude increase in $\Delta T_{\text {p }}$ (Fig. 5): a highly undesired result. For the straight lead the largest effect of the metallic shield was observed on the calibration factor, while the transfer function $S(l)$ remained practically unaltered. One would assume that a shield around a lead reduces the heating for all cases. We believe that the inconsistent behavior of the shield, (i.e., heating increase or decrease depending on the lead design) is because the shield, interacting with the lead, absorbs more energy in the case of the helical lead design. This energy cannot be properly dissipated in the small $3 \mathrm{~mm}$ long contact region. Further investigations are planned to assess the effect of the shield when the shield to medium contact area is increased, up to the entire length of the lead.

Our results are limited to the chosen geometries, i.e., helical and straight lead, and cannot be applied to other lead geometries. However, we could show that the shield, which should reduce the amount of energy absorbed by the lead, increases the heating for the helical lead. Therefore, any new lead geometry needs to be carefully assessed to ensure the safety of the device under all possible scan conditions.

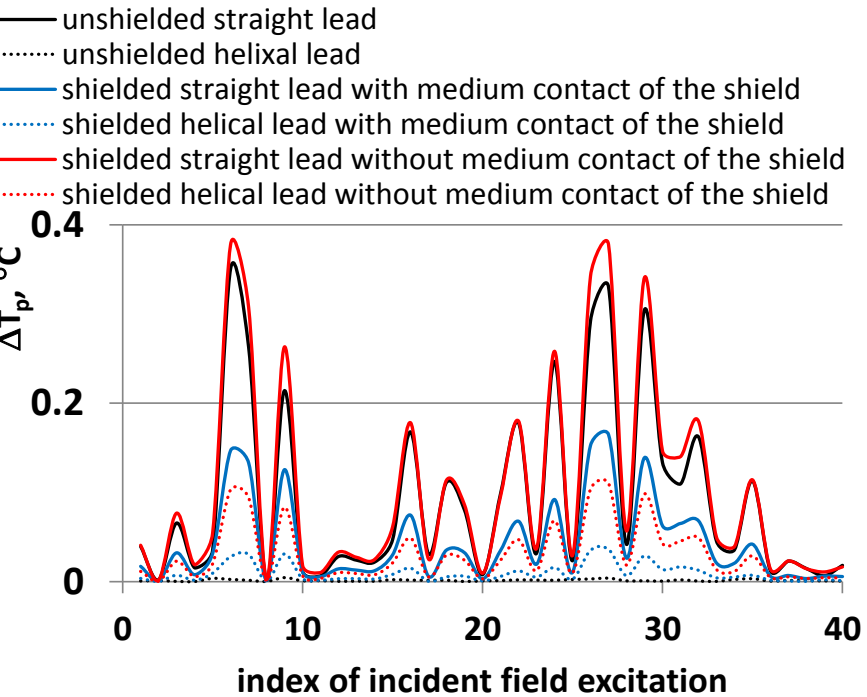

Fig. 5. $\Delta \mathrm{T}_{p}$ versus index of incident field excitation for different lead geometries and insulator $\varepsilon_{\mathrm{r}}=2.7$.

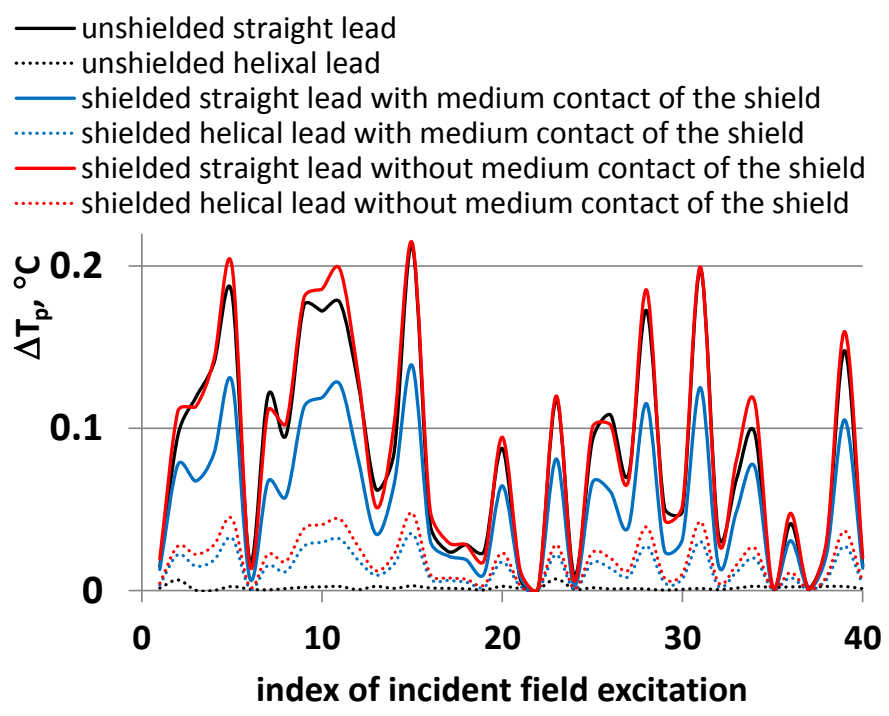

Fig. 6. $\Delta \mathrm{T}_{p}$ versus index of incident field excitation for different lead geometries and insulator $\varepsilon_{\mathrm{r}}=5.5$.

\section{DISCLAIMER}

The mention of commercial products, their sources, or their use in connection with material reported herein is not to be construed as either an actual or suggested endorsement of such products by the Department of Health and Human Services.

\section{REFERENCES}

[1] L. Panych and B. Madore, "The physics of MRI safety", Journal of Magnetic Resonance Imaging, vol. 47, no. 1, pp. 28-43, 2018.

[2] T. Song, Z. Xu, MI. Iacono, LM. Angelone, S. Rajan, "Retrospective analysis of RF heating measurements of passive medical implants", Magn Reson Med., 2018, DOI: 10.1002/mrm.27346.

[3] K. Fujimoto, LM. Angelone, E. Lucano, SS Rajan and MI. Iacono, "Radio-Frequency Safety Assessment of Stents in Blood Vessels During Magnetic Resonance Imaging", Front. Physiol. 9:1439. doi: 10.3389/fphys.2018.01439.

[4] B. Bhusal, et al., "Measurements and simulation of RF heating of implanted stereo-electroencephalography electrodes during MR scans", Magn Reson Med., vol. 80, pp.1676-1685, 2018.

[5] Technical specification ISO/TS 10974:2018, “Assessment of the safety of magnetic resonance imaging for patients with an active implantable medical device", 2018.

[6] K. Singhal and J. A. Nyenhuis, "Analysis and Design of Lead Wires with Metallic Shielding for Reduction of RF Heating during MRI for Active Implants", Proceedings of 26th Annual ISMRM Conference, 2018, p. 1454.

[7] M. Kozlov, W. Kainz, "Lead Electromagnetic Model to Evaluate RFInduced Heating of a Coax Lead: A Numerical Case Study at 128 MHz", IEEE Journal of Electromagnetics, RF and Microwaves in Medicine and Biology, vol. 2, no. 4, 2018, pp. 286-293, 2018.

[8] S. Feng, R. Qiang,W. Kainz, and J. Chen, "A technique to evaluate MRI-Induced electric fields at the ends of practical implanted lead," IEEE Trans. Microw. Theory Techn., vol. 63, no. 1, pp. 305-313, Jan. 2015 